\title{
Concentration Impairment, CTCAE
}

National Cancer Institute

\section{Source}

National Cancer Institute. Concentration Impairment, CTCAE. NCI Thesaurus. Code C143379.

A disorder characterized by a deterioration in the ability to concentrate. 